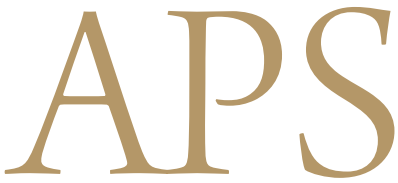

Archives of Plastic Surgery

\title{
A New Modality for Treating Congenital Melanocytic Nevus: "Cogwheel Pattern" Serial Excision Method
}

\author{
Hyun Gu Kang, Myong Chul Park, Dong Ha Park \\ Department of Plastic and Reconstructive Surgery, Ajou University Hospital, Ajou University School of Medicine, Suwon, Korea
}

Congenital melanocytic nevus consists of congregations of nevomelanocytes. It is found in approximately $1 \%$ of new born infants. Congenital melanocytic nevus needs to be excised before it transforms into a malignant lesion. Many strategies have been attempted for the removal and reconstruction of the nevus. Serial excision enables wound closure to be accomplished with a shorter scar than if the original lesion was elliptically excised in a single stage and reorientation of the scar closer to the relaxed skin lines. The routine utilization of an elliptical serial excision as a standard method of closure often leads to the formation of elongated scars and waste of skin. The "Cogwheel pattern" serial excision is a new technique for reducing the size of the nevus efficiently. Reducing the final scar length, distributing the tension over many directions, and having the chance of decrease in operation numbers are ultimately achieved with the use of the "Cogwheel pattern" serial excision.

Keywords Pigmented nevus / Wound closure techniques / Surgical flaps
Correspondence: Dong Ha Park

Department of Plastic and

Reconstructive Surgery, Ajou

University Hospital, Ajou University

School of Medicine, 164 Worldcup-ro,

Yeongtong-gu, Suwon 443-721, Korea

Tel: +82-31-219-5614

Fax: +82-31-219-5610

E-mail: growhand@hanmail.net

Received: 1 Apr $2014 \bullet$ Revised: 23 Apr $2014 \bullet$ Accepted: 24 Apr 2014

pISSN: 2234-6163 • elSSN: 2234-6171 • http://dx.doi.org/10.5999/aps.2014.41.4.418• Arch Plast Surg 2014;41:418-420

No potential conflict of interest relevant to this article was reported.

\section{INTRODUCTION}

Congenital melanocytic nevus consists of congregations of nevomelanocytes. Congenital melanocytic nevus is usually classified according to its predicted largest diameter in adulthood. Small nevi are up to $1.5 \mathrm{~cm}$, medium are 1.5 to $19.9 \mathrm{~cm}$, and large nevi are those with an estimated diameter of more than 20 $\mathrm{cm}$. Giant nevi are $50 \mathrm{~cm}$ or larger. Although nonsurgical options have been advocated for the treatment of nevus, such as derma- brasion and laser ablation, these procedures may decrease the burden of nevus cells but do not achieve complete removal of all cells [1]. Therefore, direct excision of the nevus remains the mainstay of treatment [1]. There are numerous surgi- cal options to cover the skin defect after excision of a nevus [1]. The simplest option involves serial excision and direct closure of the defect in stages, while other options include skin grafting and tissue expansion [1]. Serial excision is the excision of a lesion in more than one stage. The inherent viscoelastic properties of skin are used, allowing the skin to stretch over time. These conventional techniques enable wound closure to be accomplished with a shorter scar than if the original lesion was elliptically excised in a single stage and reorientation of the scar closer to the relaxed skin lines. However, with each stage of the serial excision there is some recoil. Herein, we introduce the "Cogwheel pattern" excision method, a newly developed method of serial excision. 


\section{Fig. 1. "Cogwheel pattern" design of serial excision}

(A) Congenital melanocytic nevus on the left chest wall. (B) "Cogwheel pattern" was designed for treatment of the congenital melanocytic nevus. (C) Congenital melanocytic nevus was partially excised in the superficial fascial layer. (D) At three weeks postoperatively, the width of the nevus was decreased by more than 50 percent.
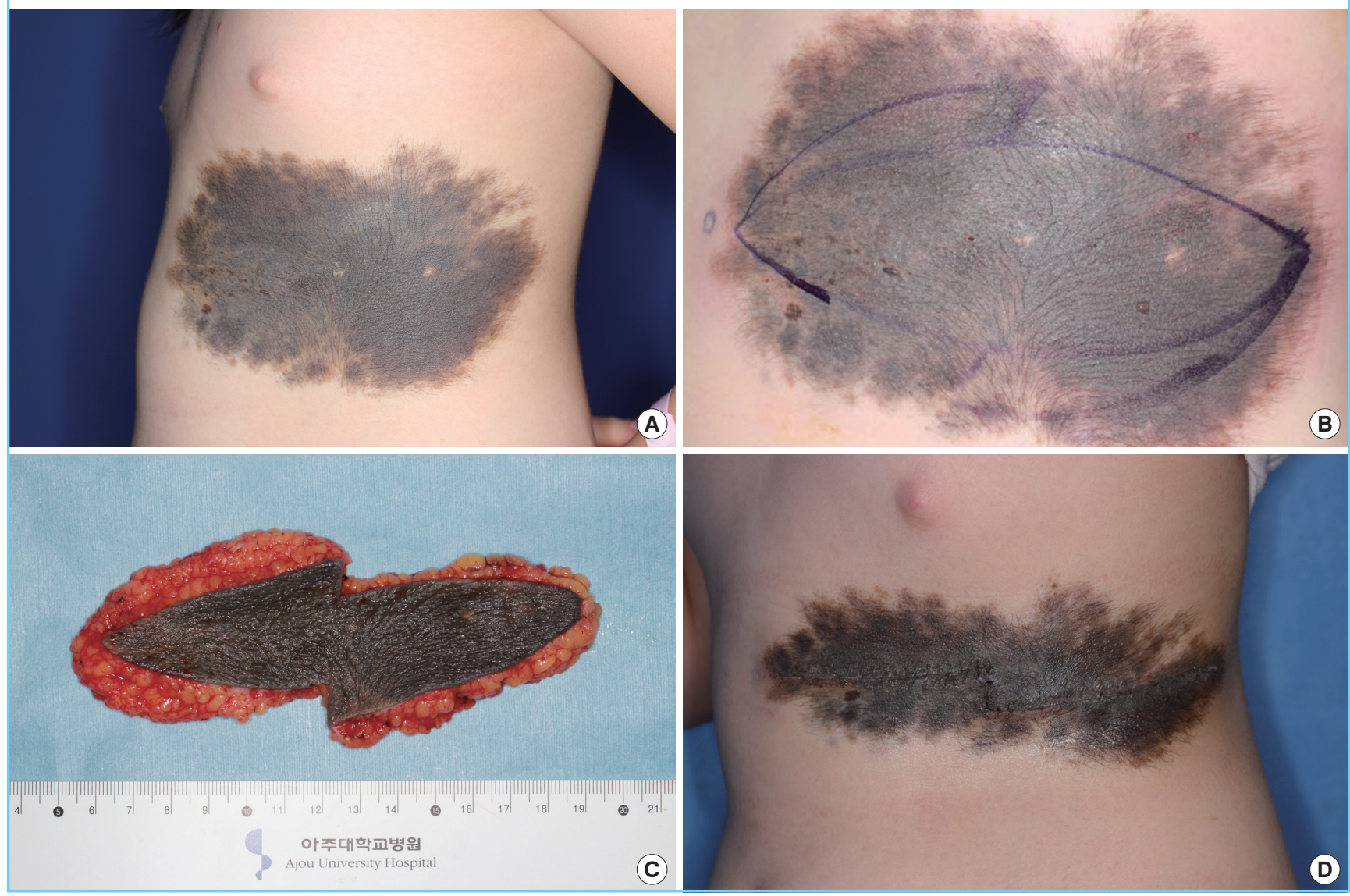

\section{IDEA}

A 9-year-old female patient presented with a congenital melanocytic nevus of the left chest wall. The patient had never undergone laser treatment or surgery. On physical examination, a 19 $\mathrm{cm} \times 9 \mathrm{~cm}$ sized nevus was detected on the left chest wall (Fig. 1). Irregular margins with hair and black to brown discoloration of the overlying skin were observed. An incision was made on the congenital melanocytic nevus using the "Cogwheel pattern" design (Fig. 1). The nevus was excised partially with \#15 blade and Bovie electrocoagulator. Considering that the congenital pigmented nevus cells are present in the subcutaneous fat layer, the nevus was excised in the superficial fascial layer (Fig. 1). After achieving complete hemostasis and undermining, the superficial fascial layer was closed with an absorbable suture and the "Cogwheel pattern" incision was closed in layers (Fig. 1). There were no complications such as infection, hematoma, distortion, or unwanted scar formation. The post-excision status was better than that after application of the conventional method. The length of the scar and dog ear deformity were decreased. The "Cogwheel pattern" incision provided the benefits of the Zplasty incision. Tensile forces were distributed in many directions. The width of the nevus was decreased by more than 50 percent postoperatively. The shape of the nevus post-excision was elliptical, and therefore it would be easier to perform the second serial excision and to finally achieve scar reduction.

\section{DISCUSSION}

Congenital melanocytic nevus is found in approximately $1 \%$ of new born infants [2]. It needs to be excised before it transforms into a malignant lesion. The routine utilization of an elliptical serial excision as a standard way of closure often leads to the formation of elongated scars and waste of skin. Using the conventional method, the normal skin is sacrificed when excising the 'dog ear deformity', resulting in an unwanted elongated scar. With the conventional method, the central portion of the nevus is excised more than the lateral portion of the nevus. Therefore, 


\section{Fig. 2. Congenital melanocytic nevus with a geographic shape}

(A) Nevus width is reduced more at the central portion after the conventional method. (B) After the "Cogwheel pattern" serial excision, the remnant nevus is elliptical in shape. Normal skin is sacrificed when excising the 'dog ear deformity', resulting in an unwanted elongated scar.

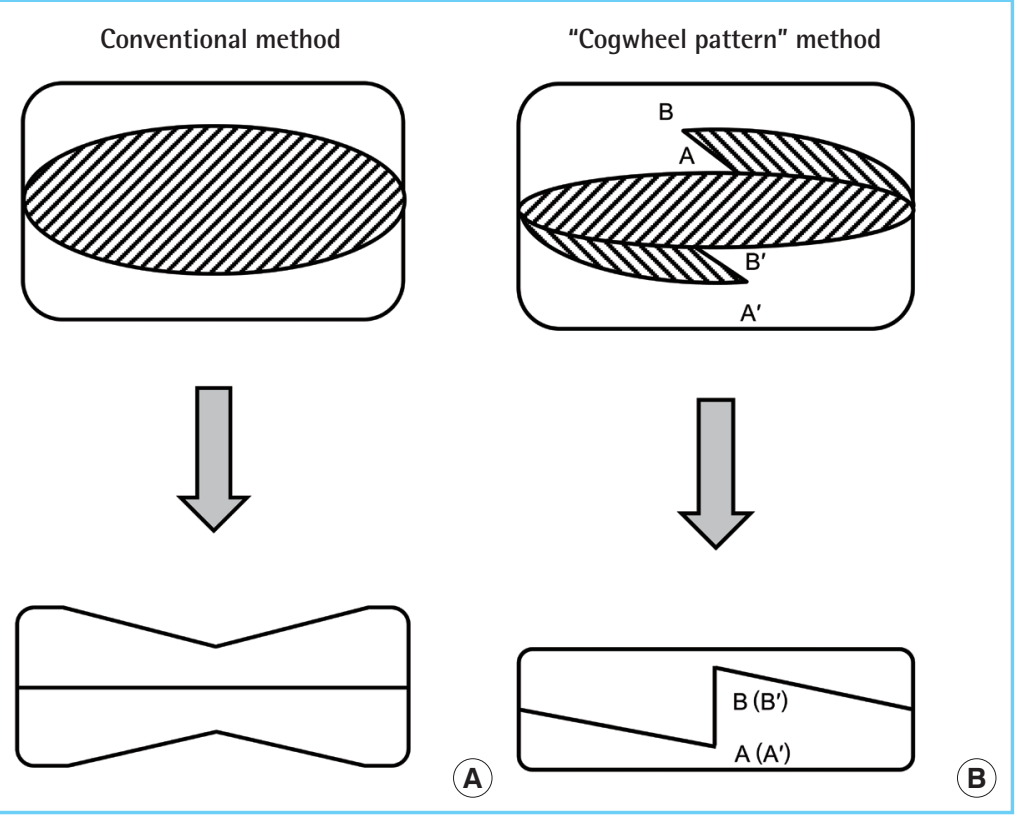

a longer incision is needed during the second excision (Fig. 2).

In order to minimize the length of the final scar, all incisions prior to the final excision, should be placed within the area to be excised [3]. Intralesional excision is particularly important for pigmented lesions as it avoids any 'spill over' of the pigment onto the normal skin [3]. Pigment cells within a nevus can migrate or be transported across a suture line through suture tracts [3]. Stretching of the scar between excisions ('stretch back') [4] will reduce the optimal effect of repairing normal skin, and thus reduce the efficiency of the technique.

Serial excision with the "Cogwheel pattern" method is simple and it has many advantages. It can reduce the final scar length by avoiding the dog ear deformity. All incisions are placed in the nevus. By suturing the superficial fascial layer and Z-plasty, it can prevent the occurrence of the 'stretch back' [4] phenomenon. It can also have the possibility to decrease numbers of operation and lower the cost of the entire treatment. All of these benefits are ultimately obtained with the "Cogwheel pattern" serial excision. This new technique can be applied for treatment of nevus sebaceus, large skin scar, or any other nevi.

\section{REFERENCES}

1. Arneja JS, Gosain AK. Giant congenital melanocytic nevi of the trunk and an algorithm for treatment. J Craniofac Surg 2005; 16:886-93.

2. Michel JL, Chalencon F, Gentil-Perret A, et al. Congenital pigmented nevus: prognosis and therapeutic possibilities. Arch Pediatr 1999;6:211-7.

3. Altchek ED. A technical consideration in the serial excision of a nevus. Plast Reconstr Surg 1980;66:849-50.

4. Nordstrom RE. "Stretch-back" in scalp reductions for male pattern baldness. Plast Reconstr Surg 1984;73:422-6. 